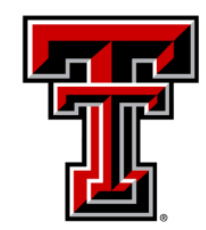

TEXAS TECH UNIVERSITY

Libraries"

\title{
ASYMMETRIC CONDUCTION IN BIOLOGICAL NANOPORES CREATED BY HIGH- INTENSITY, NANOSECOND PULSING: INFERENCE ON INTERNAL CHARGE LINING THE MEMBRANE BASED ON A MODEL STUDY
}

\section{The Texas Tech community has made this publication openly available. Please share how this access benefits you. Your story matters to us.}

\begin{tabular}{|l|l|}
\hline Citation & $\begin{array}{l}\text { R. P. Joshi and H. Qiu, "Asymmetric Conduction in Biological } \\
\text { Nanopores Created by High-Intensity, Nanosecond Pulsing: } \\
\text { Inference on Internal Charge Lining the Membrane Based on a } \\
\text { Model Study," Journal of Applied Physics 118, 094701 (2015). } \\
\text { https://doi.org/10.1063/1.4929808 }\end{array}$ \\
\hline Citable Link & $\underline{\text { https://hdl.handle.net/2346/86896 }}$ \\
\hline Terms of Use & $\begin{array}{l}\text { This article may be downloaded for personal use only. Any other } \\
\text { use requires prior permission of the author and AIP Publishing. }\end{array}$ \\
\hline
\end{tabular}




\section{Asymmetric conduction in biological nanopores created by high-intensity, nanosecond pulsing: Inference on internal charge lining the membrane based on a model study}

Cite as: J. Appl. Phys. 118, 094701 (2015); https://doi.org/10.1063/1.4929808

Submitted: 25 February 2015 . Accepted: 15 August 2015. Published Online: 01 September 2015

R. P. Joshi, and H. Qiu
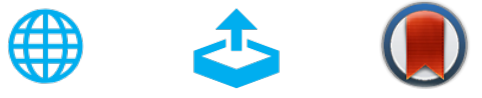

\section{ARTICLES YOU MAY BE INTERESTED IN}

Ion current rectification inversion in conic nanopores: Nonequilibrium ion transport biased by ion selectivity and spatial asymmetry

The Journal of Chemical Physics 138, 044706 (2013); https://doi.org/10.1063/1.4776216

Challenge us. What are your needs for periodic signal detection?

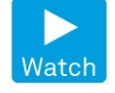

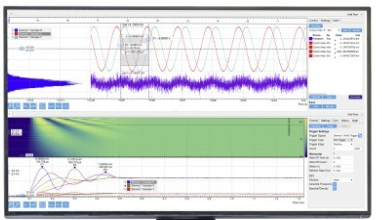

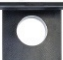

Zurich Instruments 


\title{
Asymmetric conduction in biological nanopores created by high-intensity, nanosecond pulsing: Inference on internal charge lining the membrane based on a model study
}

\author{
R. P. Joshi ${ }^{1, a)}$ and H. Qiu ${ }^{2}$ \\ ${ }^{1}$ Department of Electrical and Computer Engineering, Texas Tech University, Lubbock, Texas 79409, USA \\ ${ }^{2}$ Department of Electronic Engineering Technology, Fort Valley State University, Fort Valley, Georgia 31030 , \\ USA
}

(Received 25 February 2015; accepted 15 August 2015; published online 1 September 2015)

\begin{abstract}
Nanosecond, high-intensity electric pulses have been reported to open rectifying pores in biological cell membranes. The present goal is to qualitatively understand and analyze the experimental current-voltage $(I-V)$ data. Here, nanopore transport is probed using a numerical method and on the basis of an analytical model. Our results show that geometric asymmetry in the nanopore would not yield asymmetry in the $I-V$ characteristics. However, positive surface charge lining the pore could produce characteristics that compare well with data from patch-clamp measurements, and a value of $\sim 0.02 \mathrm{C} / \mathrm{m}^{2}$ is predicted from the numerical calculations. (C) 2015 AIP Publishing LLC.

[http://dx.doi.org/10.1063/1.4929808]
\end{abstract}

\section{INTRODUCTION}

Cells of all life forms are surrounded by membranes, consisting of lipids and proteins, which serve as a hub in mediating numerous cellular functions. ${ }^{1}$ Cell membranes strive to maintain the ionic electrochemical gradient, which is important for the transport of nutrients and generation of electrical signals. Transport usually occurs through channel proteins, but can also be facilitated by membrane pores if they can be formed. The present contribution focusses on transport through artificially generated nanopores and seeks to understand the asymmetry that has been observed in the currentvoltage characteristics through patch-clamp measurements.

Creation of nanopores in biological cell membranes can be achieved in various ways including application of external voltages (leading to electroporation ${ }^{2}$ ), ultrasonic pressure leading to sonoporation, ${ }^{3}$ laser excitation, ${ }^{4-7}$ biochemical analytes, ${ }^{8-10}$ changes in $\mathrm{pH}^{11-13}$ temperature variations, ${ }^{14}$ and by pore-forming toxins. ${ }^{15}$ The relatively recent development of using intense electric fields $(\sim 100 \mathrm{kV} / \mathrm{cm})$ and ultrashort durations ( $\sim 10$ nanoseconds) has opened up the possibility of creating nanopores in biological membranes. Such high intensity pulsing has been shown to be useful for a variety of applications ranging from reversible membrane electroporation, ${ }^{16-19}$ electrically triggered intra-cellular calcium release, ${ }^{20-22}$ the destruction of micro-organisms, ${ }^{16,23}$ and killing of tumor cells. ${ }^{16,24}$ Typical pore diameters have been predicted to be on the order of $1.6 \mathrm{~nm}$ with about a $0.6 \mathrm{~nm}$ statistical spread ${ }^{17,18,25-29}$ and roughly verified by tracking the movement of fluorescent dyes through cell membranes. ${ }^{16}$ The transport of ions through nanopores has been studied through patch-clamp experiments. ${ }^{30-32}$ Interestingly, the ionic transport has shown an asymmetric characteristics, similar to that observed in synthetic membranes. ${ }^{33-45}$ Hence,

\footnotetext{
a) Author to whom correspondence should be addressed. Electronic mail: ravi.joshi@ttu.edu
}

intuitively, one might expect similar transport physics, and inherent mechanisms such as asymmetric membrane charge distributions to be at work in biological nanopores as well.

Here, ionic transport through biological nanopores is evaluated both through simulations based on a continuum approach and an analytic treatment. We emphasize that the inherent physics and the ongoing processes are complex, and differences in the present bio-system (as compared with the often studied synthetic membranes) include: (a) Typical channel lengths of $\sim 5 \mathrm{~nm}$, which are orders of magnitude smaller than that in typical synthetic pores. This is an important difference since reports in the literature on asymmetric nanopore currents seem to conclude that no observable rectification can occur at these short channel lengths and that long pores are indispensable. ${ }^{46}$ In the different context of ion-channels, rectification has, however, been reported as a result of electrostatic interactions between proteins and the flowing charges. ${ }^{47,48}$ In any event, due to the short (nanoscale) pore lengths, spatial variations along the electric field within the nanopore can be expected to be quite minimal. (b) In addition, the molecules lining the pore are much less rigid and could allow for size and/or shape changes due to either ion bombardment from the electroosmotic flows and/or the local generation of electric stress tensors associated with the heterogeneous permittivities. (c) The behavior of biomembranes, unlike that in artificial and/or inorganic materials, is more complex. For instance, the bio-membrane thickness can vary locally or have changes in their rigidity moduli, ${ }^{49,50}$ some of which can arise from chemically induced changes in the cytoskeleton. Such adaptive phenomena are absent in synthetic membranes and inorganic materials. (d) The formation of pores can create internal stress within the membrane as the lipid bilayer molecules are displaced. This can then impact mechano-sensitive channels embedded in biological membranes and contribute to current conduction. $^{51,52}$ Such mechano-sensitive channels and pathways are absent in synthetic/inorganic membranes. (e) Finally, 
calcium, which is a secondary messenger in biosystems, can play a key role in cell biochemistry. It can be released from the endoplasmic reticulum (within cells) upon cellular stimulation by the external electric fields. ${ }^{20,22}$ Capacitive entry of calcium into cells from the extracellular medium is also possible and can lead to significant transient increase in concentration. Regardless of exact source (intra- or extracellular) of the calcium or their relative contributions, the presence of this ion can be expected in electroporation environments. Calcium has been observed to bind to nanopores, thereby modulating their internal surface charge ${ }^{53}$ and could then affect ionic transport through biological pores.

This contribution attempts to qualitatively understand and analyze the experimental current-voltage $(I-\mathrm{V})$ data that have been obtained from pulsing biological cells. ${ }^{30}$ The goal is to understand the physics behind the nonlinear, asymmetric current-voltage characteristics of conduction in nanopores. The analyses presented also reveals the necessity of a positive surface charge lining the nanopore. Basically then, a simulation approach for electroosmotic flow is used here that is similar to the previous models. ${ }^{54}$ It couples the NernstPlanck equations for ionic concentrations, the Poisson equation, and Navier-Stokes equation for the flow. An analytical approach is also used, which can facilitate the extraction of membrane charge polarity and magnitude. For completeness, it may be mentioned that alternate methods for extracting the fixed charge in biological channels have also been proposed. ${ }^{55,56}$ In these methods, the coupled Poisson-NernstPlanck (PNP) methods are used to define current transport in ion channels, and the fixed channel charge density is then extracted by solving the inverse problem. However, unless care is exercised, such approaches can have several ill-posed steps as has been discussed by Wolansky and Taflia. ${ }^{57}$

\section{METHODS AND TECHNIQUE}

The PNP equations for ionic mass transport and NavierStokes description for hydrodynamic fluid flow were used to predict electrokinetic transport in the membrane nanopore. Consideration of the fluid flow (via the Navier-Stokes equations) was included because application of an external electric field drives ions in the solution, which in turn, drag the surrounding fluid leading to electroosmosis. The PNP model $^{58-62}$ was used since the assumption of a relatively small Debye length is no longer valid with reductions in the channel dimensions, and so Boltzmann-distributions can no longer be assumed. ${ }^{6,64}$ The PNP approach treats average ion distributions in the mean-field approximation, and aspects such as fluctuations, correlations, finite size of ions, and discreteness of the solvent molecules are neglected.

The simulation geometry of the bio-membrane nanopore with axial length $L(\sim 5 \mathrm{~nm})$ and a conical cross-section with radii $R_{l}$ and $R_{r}$ are shown in the schematic of Fig. 1. It shows a cylindrical system with axial $z$ and radial $r$ coordinates with the origin fixed at the center of the nanopore. It has been argued that nanopores resulting from high-intensity, nanosecond pulsing would have an asymmetric shape with $R_{l} \neq R_{r}{ }^{65}$ The simulation region is represented by the outer boundary $\mathrm{ABCDEFGH}$, the line of symmetry by $\mathrm{AH}$, while

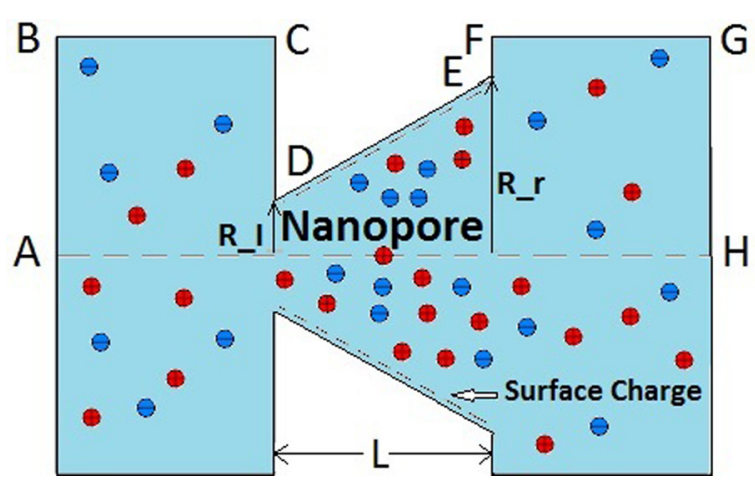

FIG. 1. Schematic of a nanopore of length $L$ with radii $R_{l}$ and $R_{r}$ connecting two identical reservoirs on either side. The surface charge density $\sigma$ is shown along the nanopore wall, which could be nonuniform. Spherical particles representing ions are also shown within the nanopore. A potential difference was assumed to be present between the planes AB and HG. The solution was taken to be $140 \mathrm{mM} \mathrm{Cs-acetate}$ at a $\mathrm{pH}$ of 7.2 in keeping with experiments. $^{30}$

the line segments, $\mathrm{AB}, \mathrm{BC}, \mathrm{FG}$, and $\mathrm{GH}$, represent the regions in the reservoirs. The ends of the pore were assumed to be connected to two fluid reservoirs. The wall of the nanopore (segment DE in Figure 1) would bear a surface charge density, $\sigma$, and in the present simulations different values were assigned to obtain the $I-V$ predictions. No charge was assigned to the reservoir walls. Bulk ionic concentrations were maintained as boundary conditions far away (five times the Debye length) from the conical nanopore. The Debye length $\left(L_{D}\right)$ is ${ }^{66} L_{D}=\left[\left(\varepsilon_{0} \varepsilon_{\mathrm{w}} k_{B} T\right) /\left(2 \mathrm{q}^{2} N_{A} 10^{3} I_{c}\right)\right]^{1 / 2}$, where $\varepsilon_{0}$ denotes the permittivity of vacuum, $\varepsilon_{w}$ is the relative dielectric constant of water, $k_{B}$ is the Boltzmann constant, $T$ is the absolute temperature, $q$ is the elementary charge, $N_{A}$ is the Avogadro number, and $I_{c}=\sum_{i= \pm} \nu_{i}^{2} \mathrm{c}_{i}(\infty) / 2$ defines the ionic strength of the electrolyte. The permittivity within the pore $\left(=\varepsilon_{\mathrm{w}} \varepsilon_{0}\right)$ was taken to be $73 \varepsilon_{0}$ (Refs. 67 and 68 ) because the reduced dimensionality and confinement in nanopores suppress screening by the water molecules. For $c_{-}(\infty)=c_{+}(\infty)=\sim 0.15 M$, the Debye length $L_{D}$ is $\sim 0.7 \mathrm{~nm}$ at $298 \mathrm{~K}$.

The fluid motion was taken to be governed by the following continuity and Stokes equations:

$$
\begin{gathered}
\boldsymbol{\nabla} \cdot \mathbf{u}=0 \\
-\nabla p+\mu \boldsymbol{\nabla}^{2} \mathbf{u}-F \nu_{1} c_{1} \mathbf{\nabla} \Phi-F \nu_{2} c_{2} \nabla \Phi=0
\end{gathered}
$$

where $\mathbf{u}=u \mathbf{a}_{r}+v \mathbf{a}_{z}$ denotes the fluid velocity, with $\mathbf{a}_{r}$ and $\mathbf{a}_{z}$ being the unit vectors along the radial and longitudinal z-directions, respectively, and $u$ and $v$ are the scalar speeds. Also, $p$ is the pressure; $\Phi$ is the electric potential; $c_{1}$ and $c_{2}$ are the molar concentrations of cations and anions, respectively, and $F$ is the Faraday constant. In keeping with patchclamp experiments, ${ }^{30}$ an aqueous medium with a $\mathrm{pH}$ of 7.2 consisting of $140 \mathrm{mM}$ of Cs-acetate was assumed. A boundary condition of $u=v=0$ was specified on segments $\mathrm{CD}$ and EF of Figure 1. A normal flow with $p=0$ was applied to the planes of $\mathrm{AB}$ and $\mathrm{GH}$. The normal ionic flux was taken to be zero at segments $\mathrm{CD}$ and EF. Finally, the potentials at the two boundaries $\mathrm{AB}$ and $\mathrm{GH}$ were taken to be $\mathrm{V}_{\text {bias }}$ and 0 , respectively, with $\mathrm{V}_{\text {bias }}$ being the externally applied bias. 
The ionic mass transport model included the NP equations for the concentration of each ionic species and the Poisson equation. The flux density of each aqueous species in steady-state is given by

$$
\begin{gathered}
N_{i}=\boldsymbol{u} c_{1}-D_{i} \mathbf{\nabla} c_{i}-\left[\nu_{i} D_{i} q /\left(k_{B} T\right)\right] c_{i} \mathbf{\nabla} \Phi, \\
\text { with } \mathbf{\nabla} N_{i}=0,
\end{gathered}
$$

where $D_{i}$ is the diffusion coefficient for the $i$ th ionic species. Finally, the Poisson's equation for the potential $\Phi$ in this case is

$$
-\varepsilon \boldsymbol{\nabla}^{2} \Phi=\nu_{1} c_{1}+\nu_{2} c_{2}
$$

where $\varepsilon$ is the solvent permittivity.

The above system of coupled equations (1)-(4) was solved using the commercial finite-element software package, COMSOL Multiphysics 4.3b (www.comsol.com). At the charged walls, a triangular mesh with $0.015 \mathrm{~nm}$ spacing was used. The meshing at the bulk reservoirs and at the nanochannel interior was decreased to the point where no current change was observed upon further mesh refining. A minimum of 30 elements were positioned within the electric double layer, adjacent to the charged wall of the nanopore.

Finally, an analytical treatment based on a reduced onedimensional PNP model has been reported recently ${ }^{66}$ for a conical channel for the steady-state $I-V$ behavior. Rectification was shown to arise from the charging of the pore walls. The current $I$ was roughly shown to equal

$$
\begin{aligned}
I(V)= & 2 \pi F D c_{\text {bulk }}\left[R_{l} R_{r} / L\right]\left(V / V_{T}\right)\left[1-\left\{\sigma /\left(12 c_{\text {bulk }} F\right)\right\}\right. \\
& \left.\times\left\{1 / R_{l}-1 / R_{r}\right\} V / V_{T}\right],
\end{aligned}
$$

where $L$ represents the pore length, and $c_{\text {bulk }}$ is the concentration of ions in the bulk [i.e., $c_{-}(\infty)$ and $c_{+}(\infty)$ ]. The above implies that for a negative charge density $\sigma$ lining the pore walls and $R_{l}<R_{r}$ (as shown in Fig. 1), the current would increase with an increasing slope for an increasing positive bias. For increasing negative voltages, however, the current would become more negative but with a decreasing slope. This trend would qualitatively be similar to a diode-curve. Conversely, for a positive charge density $\sigma$, with increasing positive bias, the current would increase but with a decreasing slope; while for increasing negative voltages, the current would become more negative with an increasing slope. Hence, the shape of the $I-V$ behavior would depend on the polarity and magnitude of the charge lining a nanopore.

\section{SIMULATION RESULTS}

The first set of simulations for the $I-V$ characteristics through the nanopore varied the radii $R_{l}$ and $R_{r}$ at the two ends. In electroporated cellular membranes, the pore radii are known to be small and typically on the order of $1 \mathrm{~nm}$. Hence, different combinations of values were chosen to lie in this range, while being at least as large as the Debye length. The left radius was kept constant at $0.8 \mathrm{~nm}$, while the radius of the opening on the right side was changed. Values

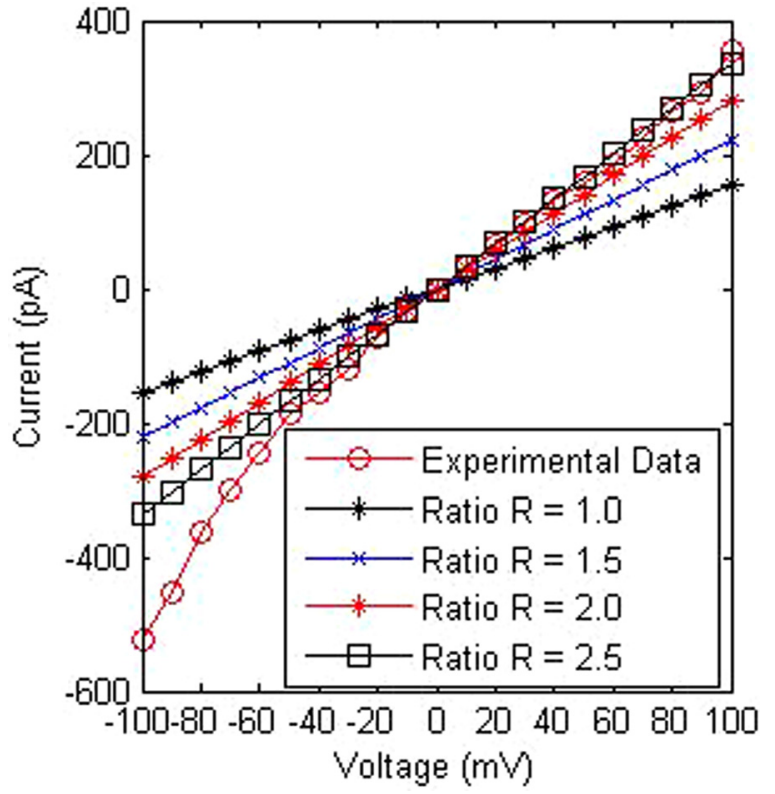

FIG. 2. Simulation results showing the current-voltage characteristics through a $5 \mathrm{~nm}$ uncharged nanopore for different aspect ratios. Experimental patch clamp data are also shown.

of the current in Fig. 2 are predicted to be higher for larger $R_{r}$ due to the wider pore opening, but despite the conical nature of the nanopore (for different aspect ratios $R=R_{r}$ l $R_{l}=1.5,2.0$, and 2.5), the $I-V$ characteristics all turn out to be nearly linear. Thus, the influence of geometry alone cannot provide nonlinearities in the $I-V$ characteristics in biological membranes. Similar conclusions have been reported for artificial membranes ${ }^{46}$ though it was suggested that long channel lengths could work to produce asymmetric $I-V$ characteristics. Figure 2 also shows experimental data that were obtained from steady-state patch-clamp experiments. ${ }^{30}$ The data reported in Fig. 1(c) of Ref. 30 were used for our comparison. However, the pore radii could not be measured and remain unknown. In any event, two points emerge from a comparison of the simulation curves with the experimental data: (i) First, the deviation between the prediction and the data seems to increase with the magnitude of the applied bias, and (ii) the experimental data are the indicative of stronger nonlinear enhancements in current with increases in the applied voltage magnitude. Furthermore, most of the $I-V$ characteristics reported in the literature on synthetic membranes do not exhibit the curvature seen in the data. However, the one-dimensional approach breaks down for large positive voltage and large currents, as was noted by Kosinska et al. ${ }^{66}$ Hence, some deviations between the predictions and the data can be expected to increase at larger bias values.

Hence, in order to better explain the experimental $I-V$ curve, the role of uniform charge lining the pore walls was examined next. Both numerical simulations and analytic calculations (as outlined in Eq. (5)) were carried out and the results were similar. In order to better match the data, the charge density values were varied as the fitting parameter. In addition, the radii $R_{l}$ and $R_{r}$ were also changed, since experimentally the values for these parameters are not 


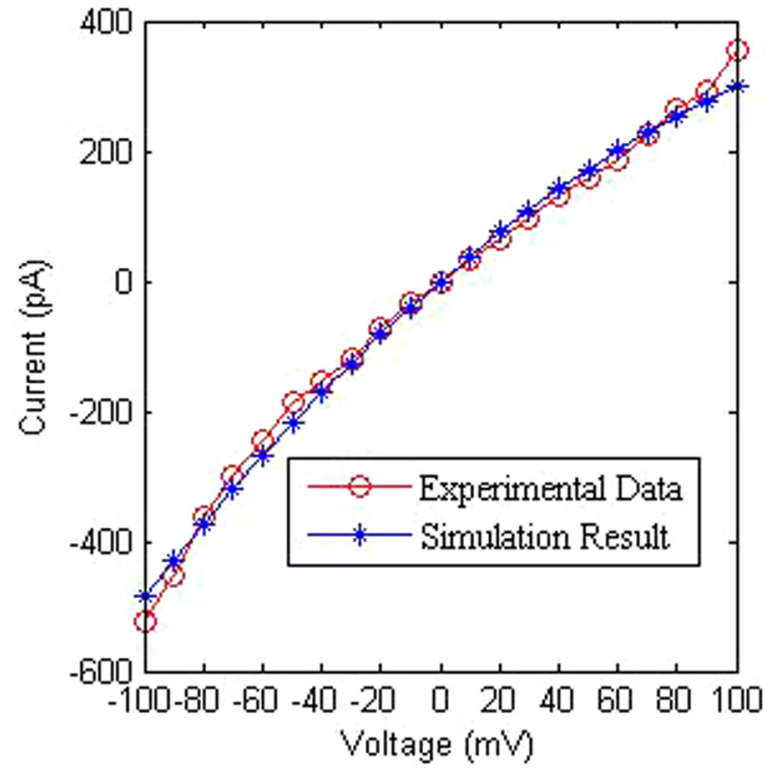

FIG. 3. Comparison between the simulation results and the experimental data showing the current-voltage characteristics through a $5 \mathrm{~nm}$ long conical nanopore. The best fit numerical prediction was obtained for $R_{l}=1 \mathrm{~nm}$, $R_{r}=2 \mathrm{~nm}$, and a charge density of $0.02 \mathrm{C} / \mathrm{m}^{2}$.

known. The best results obtained are shown in Fig. 3. Values for the surface charge $\sigma$ of $0.02 \mathrm{C} / \mathrm{m}^{2}$, and pore radii $R_{l}$ and $R_{r}$ of $1 \mathrm{~nm}$ and $2 \mathrm{~nm}$, respectively, yielded the best fit to the data. It must be mentioned here that all our numerical results for the current had to be scaled (by a factor of about 16.5) in order to bring them to match the experimental range. This is reasonable since the current in steady-state patch clamp experiments was measured over a portion of the cell membrane. Hence, contributions to ionic conduction would arise from an unknown number of nanopores distributed across the chosen membrane patch, instead of a single nanopore. Given this situation, a scaling had to be applied. The qualitative trends exhibited in the $I-V$ response can be expected to remain valid. It may also be mentioned that the charge density needed for the curve fitting is in the range reported in the literature. ${ }^{69}$ The slight deviation between the predicted currents and the data at both high voltage ends may either be due to the approximations arising from first order perturbation treatment or the neglect of a full three-dimensional details of the pore in Eq. (5). An alternate explanation may be that the analyses used here were based on rigid channel walls. While this would likely be valid for artificial membranes, the bio-channels in the present case could be subject to some deformation. For example, the constant bombardment and resulting increase in the space-charge of in-coming ions at the higher voltages might give rise to a widening of the narrow end of the pore. This would then contribute to enhanced currents as seen experimentally.

It may be mentioned that precise values or the origin of the positive charge density lining the nano-bio-pores are not known and can neither be determined experimentally nor can the pore radius be measured. Given this uncertainty, other sets of values for the pore wall charge densities and radii for the conical pore could also yield qualitative trends in keeping with the experimental data on asymmetric currents. However, the presence of a positive charge lining the membrane as postulated here for bio-channels would apply and help to explain the qualitative trends in the present context. In comparison with the artificial membranes, this predicted polarity is different. The possibility of generating $\mathrm{Ca}^{2+}$ ions, ${ }^{20,22}$ which could flow through the nanopore and bind onto inner membrane sites as has been observed, ${ }^{70}$ might be one plausible source of positive charge. These cations could come from the endoplasmic reticulum upon cellular stimulation by the external electric fields. ${ }^{22}$ Another aspect in these biological pores could be the mechanism of sodium-ion binding in which the $\mathrm{Na}^{+}$ions lie near the lipid headgroups and spend considerable time in this vicinity as reported in the literature. ${ }^{71,72}$ In a multi-ion solution, the strongest nonlinearity would arise when the attaching species had the highest concentrations. Furthermore, divalent ions such as $\mathrm{Ca}^{2+}$ would be favored to lie within the pore region over $\mathrm{Na}^{+}$(or other alkali metal) ions. The physics for this outcome is well understood and roughly arises from the following consideration. $\mathrm{Ca}^{2+}$ ions would preferentially accumulate over $\mathrm{Na}^{+}$because one divalent $\mathrm{Ca}^{2+}$ provides the same amount of charge as two $\mathrm{Na}^{+}$in about one half the ionic volume. The crowding of charges associated with the charge-space competition mechanism ${ }^{73,74}$ would produce the selectivity for $\mathrm{Ca}^{2+}$.

\section{CONCLUSIONS}

The phenomenon of electroporation of biological cell membranes finds many applications including intracellular delivery of drugs, plasmids, and in cancer therapy. From the present perspective, the nanopores afford simple biological systems for the study of electrical transport and conduction characteristics, as opposed to other more complicated biological entities such as ion-channels.

Ionic transport through biological nanopores was studied based on a continuum approach and on the basis of an analytical PNP model. The typical channel length of $\sim 5 \mathrm{~nm}$ is orders of magnitude smaller than those that typically apply to synthetic nanopores, and so spatial variations of the electric field within the nanopore can be expected to be quite minimal. Our results showed that geometric asymmetry in the nanopore would not yield asymmetry in the $I-V$ characteristics. However, positive surface charge lining the pore was shown to produce numerical results that compared well with data from patch-clamp measurements. A fairly large positive density was obtained by fitting to the experiments. This could be due to the presence of $\mathrm{Ca}^{2+}$ and would arise from charge-space competition mechanism. Further experimental studies to probe and quantify the charge state, perhaps by looking at signature shifts in laser absorption with applied polarity, would be needed. It must also be stressed that the pore radii are not measured experimentally, and so slightly different combinations of the pore aspect ratio, or surface charge might also match the data. Regardless of the precise parameter values, however, the general trend and the qualitative physics should continue to hold. Some reports in the 
literature on asymmetric nanopore currents seem to conclude that no observable rectification can occur at short channel lengths and that long pores (on the order of microns) are indispensable. ${ }^{46}$ Our analyses negate the need for long pores. Our conclusion is in agreement with the previous reports of rectification in short ion-channels ${ }^{47,48}$ that was shown to arise from electrostatic interactions between the flowing charges and channel proteins. The present model would also explain other possibilities such as dynamic current fluctuations as have experimentally been observed, ${ }^{75}$ due to dynamic attachment-detachment processes of cations at the pore inner surface. However, such time-dependent dynamics are beyond the present scope.

\section{ACKNOWLEDGMENTS}

We acknowledge useful and stimulating discussions with A. Pakhomov (Center for Bioelectrics, Old Dominion University) and S. Qian (Old Dominion University).

The authors report no conflicts of interest in this study.

${ }^{1}$ For example, D. Voet and J. G. Voet, Biochemistry (John Wiley \& Sons, New York, 2004).

${ }^{2}$ S. Mehier-Humbert and R. H. Guy, Adv. Drug Delivery Rev. 57, 733 (2005).

${ }^{3}$ T. Kodama, Y. Tomita, K. I. Koshiyama, and M. J. K. Blomley, Ultrasound Med. Biol. 32, 905 (2006).

${ }^{4}$ H. Schneckenburger, A. Hendinger, R. Sailer, W. S. L. Strauss, and M. Schmitt, J. Biomed. Opt. 7, 410 (2002).

${ }^{5}$ Y. Shirahata, N. Ohkohchi, H. Itagak, and S. Satomi, Invest. Med. 49, 184 (2001).

${ }^{6}$ S. K. Mohanty, M. Sharma, and P. K. Gupta, Biotechnol. Lett. 25, 895 (2003).

${ }^{7}$ U. K. Tirlapur and K. Konig, Nature (London) 418, 290 (2002).

${ }^{8}$ I. Vlassiouk, A. Krasnoslobodtsev, S. Smirnov, and M. Germann, Langmuir 20, 9913 (2004).

${ }^{9}$ F. Rios and S. Smirnov, ACS Appl. Mater. Interfaces 1, 768 (2009).

${ }^{10}$ D. Wandera, S. R. Wickramasinghe, and S. M. Husson, J. Membr. Sci. 357, 6 (2010).

${ }^{11}$ L. Ying, P. Wang, E. T. Kang, and K. G. Neoh, Macromolecules 35, 673 (2002).

${ }^{12}$ R. Casasus, E. Climent, M. D. Marcos, R. Martinez-Martinez, F. Sanceno, J. Soto, P. Amoros, J. Cano, and E. J. Ruiz, Am. Chem. Soc. 130, 1903 (2008).

${ }^{13}$ T. D. Nguyen, K. C. F. Leung, M. Liong, C. D. Pentecost, J. F. Stoddart, and J. I. Zink, Org. Lett. 8, 3363 (2006).

${ }^{14}$ J. H. Jang and I. In, Chem. Lett. 39, 1190 (2010).

${ }^{15}$ R. J. C. Gilbert, Cell. Mol. Life Sci. 59, 832 (2002).

${ }^{16}$ A. M. Bowman, O. M. Nesin, O. N. Pakhomova, and A. G. Pakhomov, J. Membr. Biol. 236, 15 (2010).

${ }^{17}$ K. C. Melikov, V. A. Frolov, A. Shcherbakov, A. V. Samsonov, Y. A. Chizmadzhev, and L. V. Chernomordik, Biophys. J. 80, 1829 (2001).

${ }^{18}$ V. Zlatko, A. T. Esser, T. R. Gowrishankar, and J. C. Weaver, Phys. Rev. E 74, 021904 (2006).

${ }^{19}$ N. Chen, K. H. Schoenbach, J. F. Kolb, R. J. Swanson, A. L. Garner, J. Yang, R. P. Joshi, and S. J. Beebe, Biochem. Biophys. Res. Commun. 317, 421 (2004).

${ }^{20}$ P. T. Vernier, Y. Sun, L. Marcu, S. Salemi, C. M. Craft, and M. A. Gundersen, Biochem. Biophys. Res. Commun. 310, 286 (2003).

${ }^{21}$ S. J. Beebe, P. F. Blackmore, J. White, R. P. Joshi, and K. H. Schoenbach, Physiol. Meas. 25, 1077 (2004).

${ }^{22}$ R. P. Joshi, A. Nguyen, V. Sridhara, Q. Hu, R. Nuccitelli, and K. H. Schoenbach, Phys. Rev. E 75, 041920 (2007).

${ }^{23}$ K. H. Schoenbach, R. P. Joshi, R. H. Stark, F. C. Dobbs, and S. J. Beebe, IEEE Trans. Dielectr. Electr. Insul. 7, 637 (2000).
${ }^{24}$ R. Nuccitelli, U. Pliquett, X. Chen, W. Ford, J. R. Swanson, S. J. Beebe, J. F. Kolb, and K. H. Schoenbach, Biochem. Biophys. Res. Commun. 343, 351 (2006).

${ }^{25}$ J. C. Neu and W. Krassowska, Phys. Rev. E 67, 021915 (2003).

${ }^{26}$ R. P. Joshi, Q. Hu, and K. H. Schoenbach, IEEE Trans. Dielectr. Electr. Insul. 10, 778 (2003).

${ }^{27}$ Q. Hu, R. P. Joshi, and K. H. Schoenbach, Phys. Rev. E 72, 031902 (2005).

${ }^{28}$ Q. Hu, S. Viswanadham, R. P. Joshi, K. H. Schoenbach, S. J. Beebe, and P. F. Blackmore, Phys. Rev. E 71, 031914 (2005).

${ }^{29}$ D. P. Tieleman, H. Leontiadou, A. E. Mark, and S. J. Marrink, J. Am. Chem. Soc. 125, 6382 (2003).

${ }^{30}$ A. G. Pakhomov, A. M. Bowman, B. L. Ibey, F. M. Andre, O. N. Pakhomova, and K. H. Schoenbach, Biochem. Biophys. Res. Commun. 385, 181 (2009).

${ }^{31}$ A. G. Pakhomov, R. Shevin, J. A. White, J. F. Kolb, O. N. Pakhomova, R. P. Joshi, and K. H. Schoenbach, Arch. Biochem. Biophys. 465, 109 (2007).

${ }^{32}$ B. L. Ibey, S. Xiao, K. H. Schoenbach, M. R. Murphy, and A. G. Pakhomov, Bioelectromagnetics 30, 92 (2009).

${ }^{33}$ L. A. Baker and S. P. Bird, Nat. Nanotechnol. 3, 73 (2008).

${ }^{34}$ B. Zhang, M. Wood, and H. Lee, Anal. Chem. 81, 5541 (2009).

${ }^{35}$ Z. S. Siwy, Adv. Funct. Mater. 16, 735 (2006).

${ }^{36}$ L. J. Cheng and L. J. Guo, Chem. Soc. Rev. 39, 923 (2010).

${ }^{37}$ Y. E. Korchev, C. L. Bashford, G. M. Alder, P. Y. Apel, D. T. Edmonds, A. A. Lev, K. Nandi, A. V. Zima, and C. A. Pasternak, FASEB J. 11, 600 (1997).

${ }^{38}$ E. A. Heins, Z. S. Siwy, L. A. Baker, and C. R. Martin, Nano Lett. 5, 1824 (2005).

${ }^{39}$ Z. Siwy and A. Fuliński, Am. J. Phys. 72, 567 (2004).

${ }^{40}$ J. Cervera, B. Schiedt, R. Neumann, S. Mafe, and P. Ramirez, J. Chem. Phys. 124, 104706 (2006).

${ }^{41}$ D. Constantin and Z. S. Siwy, Phys. Rev. E 76, 041202 (2007).

${ }^{42}$ M. Ali, P. Ramirez, S. Mafe, R. Neumann, and W. Ensinger, ACS Nano 3, 603 (2009).

${ }^{43}$ M. L. Kovarik, K. Zhou, and S. C. Jacobson, J. Phys. Chem. B 113, 15960 (2009).

${ }^{44}$ M. Ali, Pa. Ramirez, S. Nasir, Q. H. Nguyen, W. Ensinger, and S. Mafe, Appl. Phys. Lett. 104, 043703 (2014).

${ }^{45}$ I. Vlassiouk, S. Smimov, and Z. Siwy, ACS Nano 2, 1589 (2008).

${ }^{46}$ Q. Lin, Y. Wang, W. Guo, H. Ji, J. Xue, and Q. Ouyang, Phys. Rev. E 75, 051201 (2007).

${ }^{47}$ N. A. Simakov and M. G. Kurnikova, J. Phys. Chem. B 114, 15180 (2010).

${ }^{48}$ S. Yu. Noskov, W. Im, and B. Roux, Biophys. J. 87, 2299 (2004), and references therein.

${ }^{49}$ H. Bermúdez, D. A. Hammer, and D. E. Discher, Langmuir 20, 540 (2004).

${ }^{50}$ B. M. Discher, Y. Y. Won, D. S. Ege, J. C. M. Lee, F. S. Bates, D. E. Discher, and D. A. Hammer, Science 284, 1143 (1999).

${ }^{51}$ E. Perozo, D. M. Cortes, P. Sompornpisut, A. Kloda, and B. Martinac, Nature 418, 942 (2002).

${ }^{52}$ P. Wiggins and R. Phillips, Biophys. J. 88, 880 (2005).

${ }^{53}$ M. Ali, S. Nasir, P. Ramirez, J. Cervera, S. Mafe, and W. Ensinger, ACS Nano 6, 9247 (2012).

${ }^{54}$ Y. Ai, M. Zhang, S. W. Joo, M. A. Cheney, and S. Qian, J. Phys. Chem. C 114, 3883 (2010).

${ }^{55}$ M. Burger, R. S. Eisenberg, and H. W. Engl, SIAM J. Appl. Math. 67, 960 (2007).

${ }^{56}$ K. Arning, M. Burger, R. S. Eisenberg, H. W. Engl, and L. He, Proc. Appl. Math. Mech. 7, 1120801 (2007).

${ }^{57}$ G. Wolansky and A. Taflia, J. Chem. Phys. 133, 234113 (2010).

${ }^{58}$ S. Kang and Y. K. Suh, Microfluid. Nanofluid. 6, 461 (2009).

${ }^{59}$ S. Bera and S. Bhattacharyya, Int. J. Eng. Sci. 62, 165 (2013).

${ }^{60}$ S. E. Yalcin, S. Y. Lee, S. W. Joo, O. Baysal, and S. Qian, J. Phys. Chem. B 114, 4082 (2010).

${ }^{61}$ S. Qian, A. Wang, and J. K. Afonien, J. Colloid Interface Sci. 303, 579 (2006).

${ }^{62}$ H. Liu, S. Qian, and H. H. Bau, Biophys. J. 92, 1164 (2007).

${ }^{63} \mathrm{D}$. Li, Electrokinetics in Microfluidics (Elsevier, London, 2004).

${ }^{64}$ H. M. Park, J. S. Lee, and T. W. Kim, J. Colloid. Interface Sci. 315, 731 (2007).

${ }^{65}$ R. P. Joshi and Q. Hu, Med. Biol. Eng. Comput. 48, 837 (2010).

${ }^{66}$ I. D. Kosinska, I. Goychuk, M. Kostur, G. Schmid, and P. Hanggi, Phys. Rev. E 77, 031131 (2008) 
${ }^{67}$ M. A. Golombeck, C. H. Riedel, and O. Dossel, Biomed. Tech. (Berl). 47(Suppl. 1, Pt. 1), 253 (2002).

${ }^{68}$ R. P. Joshi, V. Sridhara, and K. H. Schoenbach, Biochem. Biophys. Res. Commun. 348, 643 (2006).

${ }^{69}$ P. Y. Apel, I. V. Blonskaya, N. V. Levkovich, and O. L. Orelovich, Petrol. Chem. 51, 555 (2011).

${ }^{70}$ R. P. Joshi and K. H. Schoenbach, Crit. Rev. Biomed. Eng. 38, 255 (2010).
${ }^{71}$ S. A. Pandit, D. Bostick, and M. L. Berkowitz, Biophys. J. 84, 3743 (2003). ${ }^{72}$ R. A. Bockmann, A. Hac, T. Heimburg, and H. Brubmuller, Biophys. J. 85, 1647 (2003).

${ }^{73}$ W. Nonner, L. Catacuzzeno, and B. Eisenberg, Biophys. J. 79, 1976 (2000).

${ }^{74}$ D. Boda, D. D. Busath, D. Henderson, and S. Sokołowski, J. Phys. Chem. B 104, 8903 (2000).

${ }^{75}$ A. G. Pakhomov, private communication (2014). 